\title{
A multi-physics and multi-scale numerical approach to microcracking and power-loss in photovoltaic modules
}

\author{
Marco Paggi*, Mauro Corrado, Maria Alejandra Rodriguez \\ Politecnico di Torino, Department of Structural, Geotechnical and Building Engineering \\ Corso Duca degli Abruzzi 24, 10129 Torino, Italy
}

\begin{abstract}
A multi-physics and multi-scale computational approach is proposed in the present work to study the evolution of microcracking in polycrystalline Silicon ( $\mathrm{Si}$ ) solar cells composing photovoltaic (PV) modules. Coupling between the elastic and the electric fields is provided according to an equivalent circuit model for the PV module where the electrically inactive area is determined from the analysis of the microcrack pattern. The structural scale of the PV laminate (the macro-model) is coupled to the scale of the polycrystals (the micro-model) using a multiscale nonlinear finite element approach where the macro-scale displacements of the Si cell borders are used as boundary conditions for the micro-model. Intergranular cracking in the $\mathrm{Si}$ cell is simulated using a nonlinear fracture mechanics cohesive zone model (CZM). A case-study shows the potentiality of the method, in particular as regards the analysis of the microcrack orientation and distribution, as well as of the effect of cracking on the electric characteristics of the PV module.
\end{abstract}

Notice: this is the author's version of a work that was accepted for publication in Composite Structures. Changes resulting from the publishing process, such as editing, structural formatting, and other quality control mechanisms may not be reflected in this document. A definitive version was published in Composite Structures, Vol. 95, January 2013, 630 638, DOI:10.1016/j.compstruct.2012.08.014

Key words: Photovoltaic module; Fracture Mechanics; Computational methods; Multi-physics; Multi-scale.

* Corresponding author. Tel. +39-011-090-4910 Fax +39-011-090-4899

Email address: marco.paggi@polito.it (Marco Paggi). 


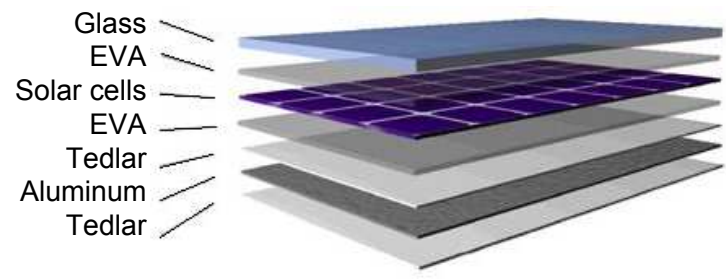

Figure 1. Sketch of the composite stack of a PV module.

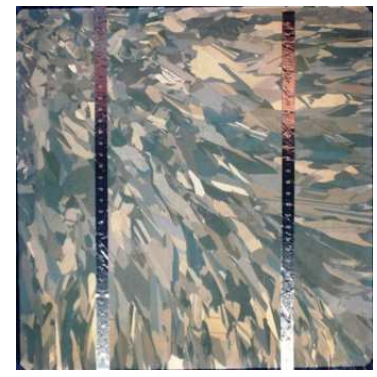

Figure 2. Microstructure of a polycrystalline Si cell. The lateral size of the cell is 125 mm.

\section{Introduction}

Photovoltaics (PV) based on Silicon ( $\mathrm{Si}$ ) semiconductors is one the most growing technology in the World for renewable, sustainable, non-polluting, widely available clean energy sources. Standard PV modules are laminates composed of a glass superstrate $4 \mathrm{~mm}$ thick, an encapsulating polymer layer (EVA) $0.5 \mathrm{~mm}$ thick, a layer of Si solar cells $0.166 \mathrm{~mm}$ thick, another layer of EVA with the same thickness as the previous one, and finally a thin multi-layered backsheet made of Tedlar/Aluminum/Tedlar $0.1 \mathrm{~mm}$ thick, see Fig.1. For more details about the geometrical and mechanical properties of these constituent materials, the reader is referred to [1]. The majority of solar cells available on the market are made of either mono or polycrystalline $\mathrm{Si}$ and are separated by a thin amount of EVA in their plane. Two main semiconductors, called busbars, connect the cells together and are placed on the upper and the lower sides of the cells. The microstructure of a polycrystalline $\mathrm{Si}$ cell is shown in Fig,2, where we note a significant elongation of the grains due to production issues.

So far, theoretical and applied research has focused on the increase of the solar energy conversion efficiency of the cells. Although efficiencies up to $40 \%$ have been reached in the laboratory using single junction GaAs and multijunction concentrators, the technology based on mono and multicrystalline Si is still the most competitive on the market due to the low price of Si semiconductor and the widely established material processing developed in the field of electronics [2].

Another important issue is the problem of durability, which, however, has received much less attention by the scientific community so far. In the 1990s, warranties 


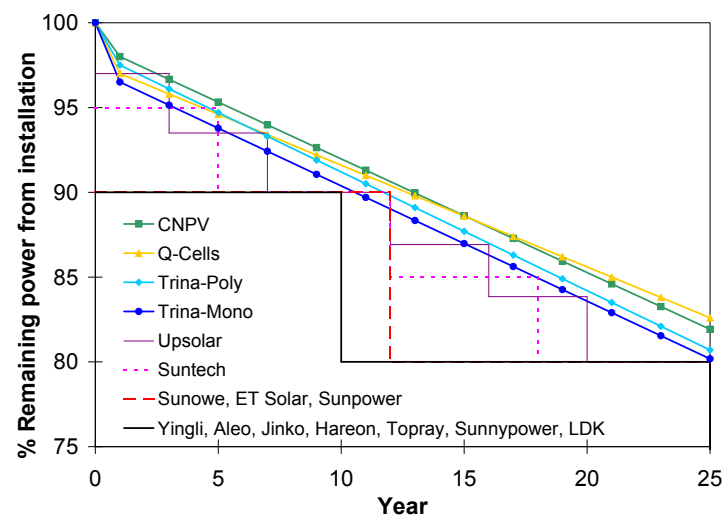

Figure 3. Comparison among warranty specifics declared by various producers of PV modules.

of PV producers allow one to replace PV modules in case of power losses larger than $10 \%$ in the first 10 years, and then larger than $20 \%$ in the next 15 years. The maximum life of PV modules is considered to be of 25 years. More recently, with more and more field data of installed modules available, a linear decreasing performance of the PV module is expected (see the comparison among various warranty specifics in Fig, 3).

The quality control of these composites is of primary concern from the industrial point of view. On the one hand, the aim is to develop new manufacturing processes able to reduce the number of cells or modules rejected by quality control [3]. On the other hand, even if all the damaged cells are theoretically discarded during manufacturing, it is impossible to avoid the occurrence of microcracking. Sources of damage in Si cells are transport, installation and use (in particular impacts, snow loads and environmental aging caused by temperature and relative humidity variations). The existing qualification standards IEC 61215 require passing of severe laboratory tests in a climate chamber. However, microcracking is not used as a quantitative indicator for the quality assessment of PV modules. Recently, Kajari-Schröder et al. [4] have analyzed microcracking resulting from snow tests and artificial aging in the laboratory using the electroluminescence technique, see Fig,4. Microcracking can lead to large electrically disconnected cell areas, with up to $16 \%$ of power-loss [5]. In addition to laboratory tests on single panels, field data published in [6] have shown that microcracked cells have a non constant current-voltage characteristics in time and an undesirable increase of the operating temperature.

The aim of the present work is to understand the phenomena leading to microcracking in Si cells and to quantify the connection between cracking and power-loss. In this study, an innovative multi-scale (multi-resolution) and multi-physics numerical method is proposed. Since Si cells operate in the presence of elastic, thermal and electric fields, a multi-field (multi-physics) perspective is considered to be essential to achieve a predictive stage of any computational model. The multi-resolution 


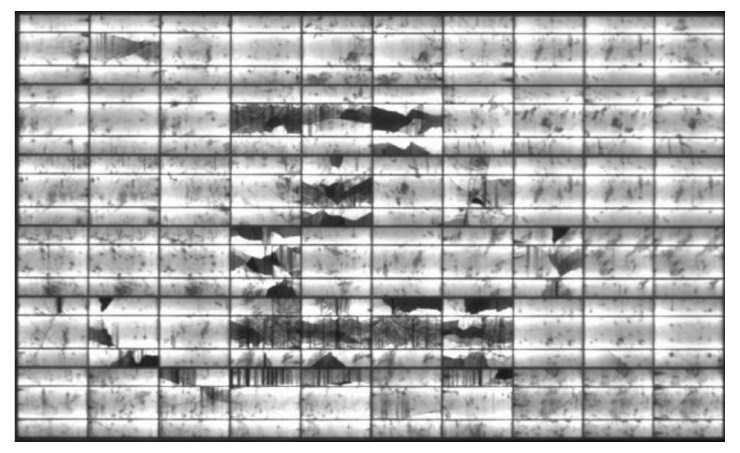

Figure 4. Electroluminescence image of a micro-cracked PV module. Dark regions are electrically inactive areas [4].

approach, on the other hand, is adopted to simplify the actual 3D problem in a simpler 2D one, where microcracking in Si cells is numerically simulated under plane stress conditions and nonlinear fracture mechanics formulations. To the knowledge of the present authors, the present model is the first proposed in the literature for the simulation of microcracking and the resulting prediction of power-loss of $\mathrm{PV}$ modules.

\section{A multi-physics approach}

The study of durability of PV modules requires the characterization of the effect of microcracking induced by mechanical loads and thermal excursions on the electric response of the solar panel. Mechanical loads are induced by vibrations and impacts during transportation, installation and use of the modules. Deformations are also induced by the night and day alternating temperature variation. Moreover, temperature affects the electric performance of the PV module, since the semiconductor differential equations present temperature dependent coefficients. Hence, to achieve a predictive stage, a computational method should account for the coupling between the elastic, the thermal and the electric fields. In other words, a multi-field (multi-physics) approach has to be pursued.

A conceptual diagram of the interplay among the various fields is shown in Fig.5. The coupling between the elastic and the thermal fields can be accounted for by the classic equations of thermoelasticity. However, in presence of cracking, a specific treatment of the partial differential equations describing heat conduction should be considered, as well as its inherent nonlinearities.

Regarding the interplay between the electric and the thermal fields, a rigorous approach should consider the partial differential equations describing the electric and magnetic fields inside the semiconductor, according to the physics of the photovoltaic effect (see Fig 6). In the present work, a simplified approach is proposed by considering an equivalent electric circuit of the cells and of the module, see 


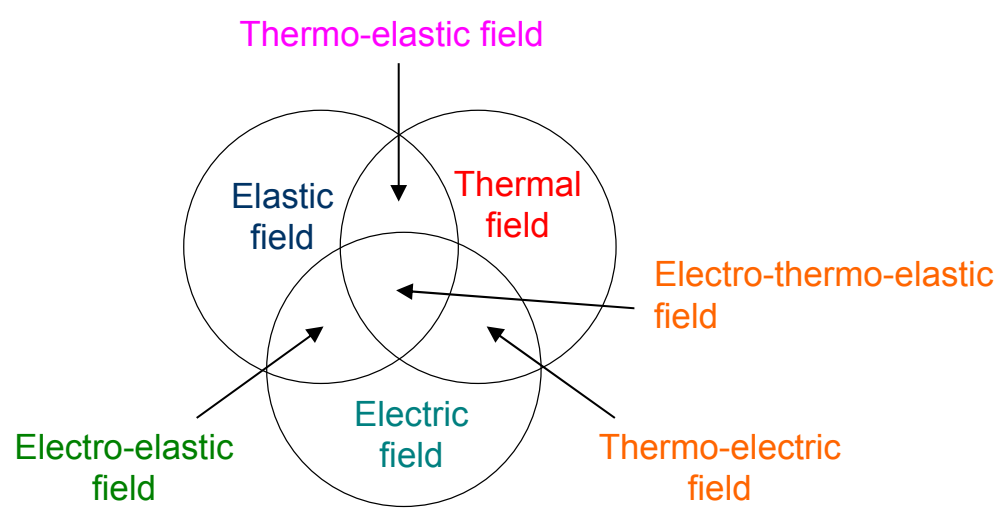

Figure 5. Interplay among the elastic, the thermal and the electric fields in PV modules.



Figure 6. Physics of a solar cell.

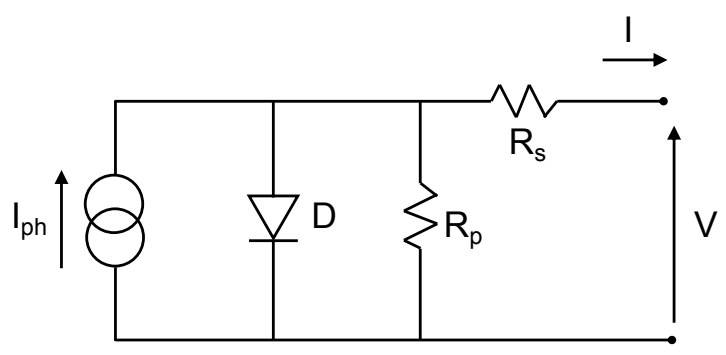

Figure 7. Single diode equivalent circuit model.

Fig.7. Although mathematically less rigorous since the interplay of the thermal and elastic fields is accounted for in a global way, this approach is considered to be particularly appealing from the industrial point of view, since it can be implemented in Matlab/Simulink or in commercial finite element (FE) software as a user-defined subroutine.

The basic idea is to consider the photovoltaic effect as the source of a photonic current $I_{\mathrm{ph}}$ due to the movement of electrons and holes in $\mathrm{Si}$ as a result of the so-called $p-n$ junction. The $p-n$ junction effect can then be modelled with one or two diodes [7. In the present study, a one single diode model is used. Series and parallel resistances complete the circuit. Particular important is the series resistance, which takes into account the bulk resistance of the semiconductor, 
contacts and interconnectors. The parallel resistance, also called shunt resistance, is used to model the effect of impurities and non idealities of the $p-n$ junction.

The equation describing the current-voltage response of a PV module with Si cells connected in series is [7]:

$$
I=I_{\mathrm{ph}}-I_{\mathrm{s}}\left\{\exp \left[\frac{e\left(V / n+I R_{\mathrm{s}}\right)}{a \kappa T}\right]-1\right\}-\frac{V / n+I R_{\mathrm{s}}}{R_{\mathrm{p}}},
$$

where $n$ is the number of cells, $I_{\mathrm{ph}}(\mathrm{A})$ is the photonic current, $I_{\mathrm{s}}(\mathrm{A})$ is the saturation current, $V(\mathrm{~V})$ is the module terminal voltage, $I(\mathrm{~A})$ is the module terminal current, $R_{\mathrm{s}}(\Omega)$ is the series resistance, $R_{\mathrm{p}}(\Omega)$ is the parallel resistance, $e=1.6 \times 10^{-19} \mathrm{C}$ is the electronic charge, $a \cong 2$ is the diode quality factor for polycrystalline $\mathrm{Si}, \kappa=1.38 \times 10^{-23} \mathrm{~J} / \mathrm{K}$ is the Boltzmann's constant, and $T(\mathrm{~K})$ is the ambient temperature.

The main quantities in Eq.(1) depend on the cell temperature $T$ [7]:

$$
\begin{aligned}
I_{\mathrm{ph}} & =I_{\mathrm{ph}}^{T=300}\left[1+k_{0}(T-300)\right], \\
I_{\mathrm{s}} & =k_{1} T^{3} \exp \left(-\frac{e V_{g}}{\kappa T}\right), \\
R_{\mathrm{s}} & =R_{\mathrm{s}}^{T=300}\left[1-k_{2}(T-300)\right], \\
R_{\mathrm{p}} & =R_{\mathrm{p}}^{T=300} \exp \left(-k_{3} T\right),
\end{aligned}
$$

where $V_{g}(\mathrm{~V})$ is the band gap voltage and the coefficients $k_{i}$ are determined from experiments [7].

From the mathematical point of view, Eq.(1) is implicit and nonlinear. Hence, for a given value of voltage $V$, its solution has to be obtained using a numerical method. Here, the Newton-Raphson method is adopted to achieve a quadratic convergence of the numerical scheme. After a suitable manipulation, Eq.(1) becomes:

$$
f(I)=I-I_{\mathrm{ph}}+I_{\mathrm{s}}\left\{\exp \left[\frac{e\left(V / n+I R_{\mathrm{s}}\right)}{a \kappa T}\right]-1\right\}+\frac{V / n+I R_{\mathrm{s}}}{R_{\mathrm{p}}}=0 .
$$

At the generic iteration $i+1$, the solution is sought from the approximation at the previous step $i$ :

$$
I_{i+1}=I_{i}-\left[\frac{\mathrm{d} f}{\mathrm{~d} I}\right]_{I_{i}}^{-1} f\left(I_{i}\right)
$$

where the derivative $\mathrm{d} f / \mathrm{d} I$ is:

$$
\frac{\mathrm{d} f}{\mathrm{~d} I}=1+I_{\mathrm{s}} \exp \left[\frac{e\left(V / n+I R_{\mathrm{s}}\right)}{a \kappa T}\right] \frac{e R_{\mathrm{s}}}{a \kappa T}+\frac{R_{\mathrm{s}}}{R_{\mathrm{p}}} .
$$


Equation (4) is iterated until convergence, i.e., until $\left|I_{i+1}-I_{i}\right|<$ tol, where tol = 0.001 is a used prescribed tolerance. The state $I_{0}=I_{\mathrm{SC}}$ is selected as the starting point for the iteration procedure.

To model the effect of cracking on the electric field, we note that the the saturation current $I_{\mathrm{s}}$ is linearly dependent on the electrically active cell area, as found in [8]. Electrically inactive cell areas are those whose electric flux directed towards the two main conductors connecting the cells (busbars) is interrupted by a crack. Examples are shown in Fig, 8 , where we note that cracks parallel to the busbar are the most dangerous ones, whereas cracks perpendicular to the busbar might have a negligible effect on the $I-V$ characteristics [5], since they do not interrupt the electron flux. Intermediate situations can occur and are related to intermediate inclinations of microcracks. According to this criterion, we introduce a damage variable for each cell defined as follows:

$$
D=\frac{A_{\text {inactive }}}{A_{\text {total }}}
$$

where $A_{\text {inactive }}$ and $A_{\text {total }}$ are, respectively, the inactive and the total cell areas. The same definition applies also to an ensemble of cells in series (a PV module). In this case the damage variable is the maximum of the damage variables of the various cells. As a result of damage, the saturation and photonic currents become:

$$
\begin{aligned}
I_{\mathrm{s}} & =I_{\mathrm{s}}^{D=0}(1-D), \\
I_{\mathrm{ph}} & =I_{\mathrm{ph}}^{D=0}(1-D) .
\end{aligned}
$$

In general, the graph of the output current $I$ as a function of voltage $V$ is almost constant and equal to the short circuit current $I_{0}=I_{\mathrm{sc}}$ for a voltage less than the open circuit voltage $V_{\text {oc }}$. For $V \rightarrow V_{\text {oc }}$, the $I-V$ curve is rounded up and the current comes to zero for $V=V_{\text {oc }}$. The output power is equal to the instantaneous product of the PV current intensity and the PV voltage, $P=I V$. Due to the shape of the $I-V$ curve, the $P-V$ characteristics is linear up to a maximum, called maximum power point, and then comes down to zero for $V=V_{\mathrm{oc}}$. The electric performance can be synthetically described by the fill factor:

$$
F F=\frac{V_{\mathrm{MP}} I_{\mathrm{MP}}}{V_{\mathrm{oc}} I_{\mathrm{sc}}}
$$

where $V_{\mathrm{MP}}$ and $I_{\mathrm{MP}}$ are the voltage and the current of the maximum power point. Hence, the power-loss due to microcracking can be estimated by comparing the fill factors of the intact module and of the damaged one. 


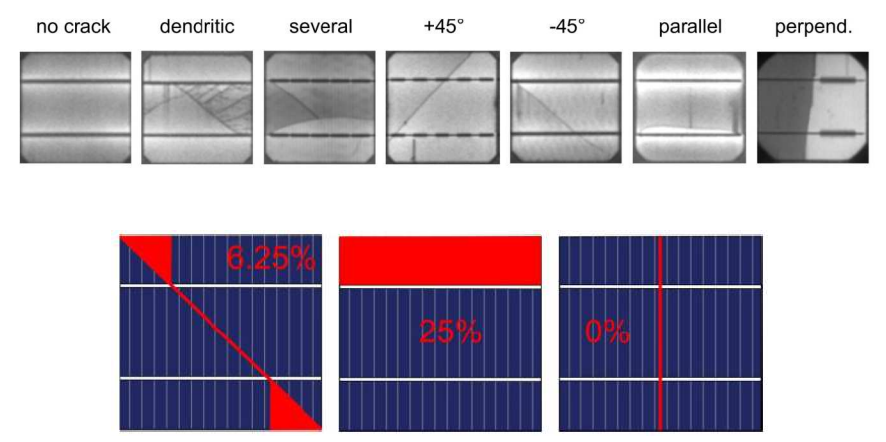

Figure 8. Representation of electrically inactive cell areas, adapted from [5].

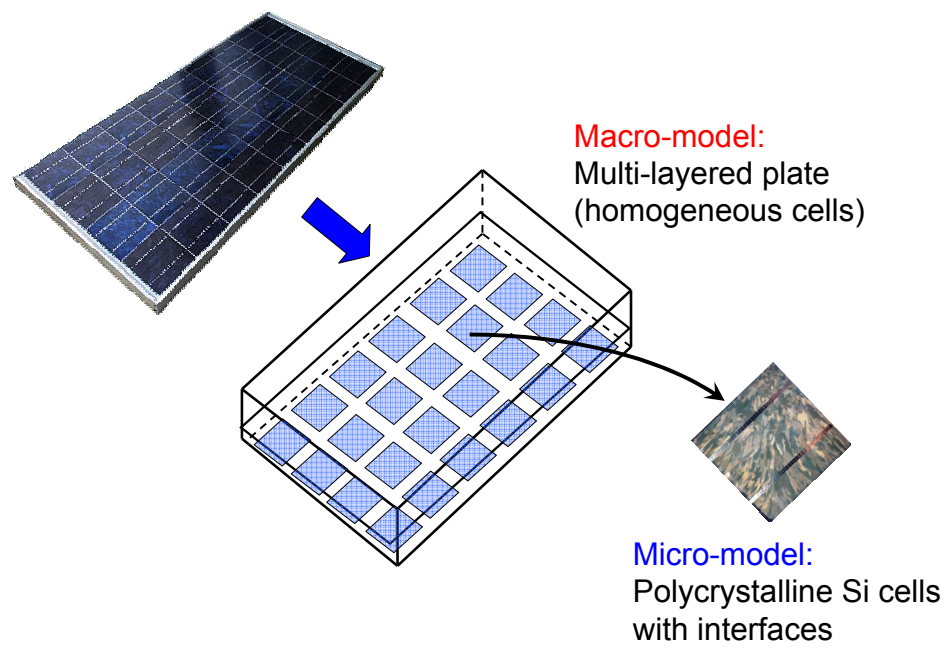

Figure 9. A real PV module and its macro- and micro-models.

\section{A multi-scale (multi-resolution) computational method}

Photovoltaic modules are laminated composites where the thickness of the various layers are very different from each other, as outlined in the Introduction. Moreover, Si cells are separated by a thin interspace of EVA and are made of a polycrystalline material whose microstructure has to be considered to predict microcracking. These complexities suggest the use of 3D methods, which are however computationally expensive. In the present study, a simplified approach to reduce the computational complexity is proposed and it is based on two levels of resolution, see Fig.9.

The macro-model consists in a laminated composite analyzed in the framework of the small-deflection theory with homogeneous Si cells. The layers are considered to be fully bonded along their interfaces. The grain boundaries in the material microstructure of Si cells and the related intergranular fracture are neglected here. The weak form for the macro-model problem is:

$$
\delta W=-\int_{\Omega} \delta(\nabla w)^{\mathrm{T}} \boldsymbol{D} \nabla w \mathrm{~d} \Omega+\int_{\Omega} q \delta w \mathrm{~d} \Omega
$$


with simple support boundary conditions on the border of the plate:

$$
w(\partial \Omega)=0
$$

where $\Omega$ and $\partial \Omega$ are, respectively, the laminate plate and its border. The variable $w$ is the transversal displacement that, together with the vector $\boldsymbol{\theta}=\left[\theta_{x}, \theta_{y}\right]^{\mathrm{T}}$ containing the nodal rotations in directions $X$ and $Y$, will represent the unknown generalized displacements of the continuum. To simulate the snow test prescribed by the standard IEC 61215 for the qualification of PV modules, a uniform transversal pressure $q$ is imposed to the upper surface of the PV module.

The operator $\boldsymbol{\nabla}$ in Eq.(9) is given by:

$$
\boldsymbol{\nabla}=\left(\frac{\partial^{2}}{\partial^{2} x}, \frac{\partial^{2}}{\partial^{2} y}, 2 \frac{\partial^{2}}{\partial x \partial y}\right)^{\mathrm{T}}
$$

The constitutive matrix $\boldsymbol{D}$ is

$$
\boldsymbol{D}=K\left[\begin{array}{ccc}
1 & \nu & 0 \\
\nu & 1 & 0 \\
0 & 0 & \frac{1-\nu}{2}
\end{array}\right]
$$

and the coefficient $K$ of the multi-layered plate depends on the layer arrangement [9]:

$$
\begin{aligned}
K & =\frac{A C-B^{2}}{A} \\
A & =\sum_{k} \frac{E_{k}}{1-\nu_{k}^{2}}\left(z_{k}-z_{k-1}\right), \\
B & =\sum_{k} \frac{E_{k}}{1-\nu_{k}^{2}} \frac{z_{k}^{2}-z_{k-1}^{2}}{2}, \\
C & =\sum_{k} \frac{E_{k}}{1-\nu_{k}^{2}} \frac{z_{k}^{3}-z_{k-1}^{3}}{3}
\end{aligned}
$$

where $k$ is the total number of layers, $z_{k}$ is the absolute value of the distance of the lower interface of the considered layer from the upper side of the plate, and $E_{k}$ and $\nu_{k}$ are the Young's modulus and the Poisson's ratio of the $k$-th layer (see Fig. 10). Since Si cells are not continuous in the plate plane, two different values of $K$ will be used for the finite elements belonging to the portions of the plates with $\mathrm{Si}$ cells and without Si cells (see also Fig 1 for a visual representation).

The FE implementation of the macro-model is done by using standard linear elastic plate elements, see Fig.10. Using quadrilater finite elements with linear shape functions, the unknown displacements and rotations are discretized with the use 

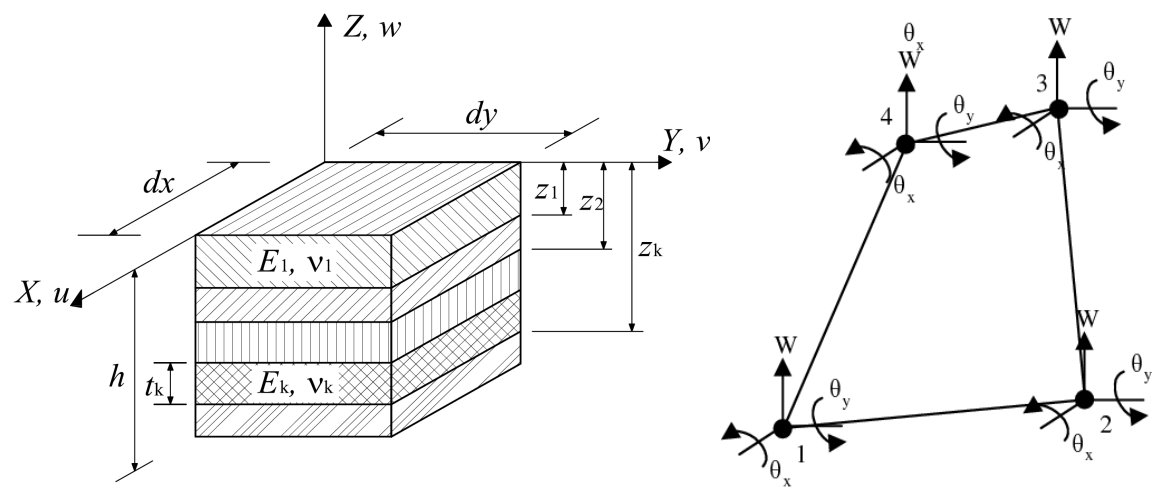

Figure 10. The plate element used in the macro-model and its degrees of freedom. of standard interpolation functions:

$$
\begin{aligned}
& w=\boldsymbol{N} \boldsymbol{\eta}, \\
& \boldsymbol{\theta}=\boldsymbol{N}_{\theta} \boldsymbol{\eta},
\end{aligned}
$$

where $\boldsymbol{\eta}=\left[w_{1}, \theta_{x 1}, \theta_{y 1}, \ldots, w_{4}, \theta_{x 4}, \theta_{y 4}\right]^{\mathrm{T}}$ and:

$$
\begin{aligned}
\boldsymbol{N} & =\left[N_{1}, 0,0, \ldots, N_{4}, 0,0\right], \\
\boldsymbol{N}_{\theta} & =\left[\begin{array}{ccccccc}
0 & N_{1} & 0 & \ldots & 0 & N_{4} & 0 \\
0 & 0 & N_{1} & \ldots & 0 & 0 & N_{4}
\end{array}\right] .
\end{aligned}
$$

Introducing these relations in the weak form (9), we have:

$$
\delta W=\delta \boldsymbol{\eta}^{\mathrm{T}}\left(-\int_{\Omega}(\boldsymbol{\nabla} \boldsymbol{N})^{\mathrm{T}} \boldsymbol{D} \boldsymbol{\nabla} \boldsymbol{N} \mathrm{d} \Omega \boldsymbol{\eta}+\int_{\Omega} \boldsymbol{N}^{\mathrm{T}} q \mathrm{~d} \Omega\right),
$$

and setting it equal to zero, for the arbitrariness of $\delta \boldsymbol{\eta}$, the standard expressions for the stiffness matrix, $\boldsymbol{K}$, and for the external load vector, $\boldsymbol{F}$, are obtained:

$$
\left(\int_{\Omega}(\boldsymbol{\nabla} \boldsymbol{N})^{\mathrm{T}} \boldsymbol{D}_{\mathrm{A}} \boldsymbol{\nabla} \boldsymbol{N} \mathrm{d} \Omega\right) \boldsymbol{\eta}=\int_{\Omega} \boldsymbol{N}^{\mathrm{T}} q \mathrm{~d} \Omega \quad \Rightarrow \quad \boldsymbol{K} \boldsymbol{\eta}=\boldsymbol{F} .
$$

The displacements $u$ (in the $x$ direction) and $v$ (in the $y$ direction) in the plane of the cells are computed from the nodal rotations as follows:

$$
\begin{aligned}
& u=\theta_{y} z, \\
& v=-\theta_{x} z,
\end{aligned}
$$

where $z=-1.953 \mathrm{~mm}$ is the distance from the center of mass of the cross-section to the Si cell plane.

It is notable to remark that these displacements impose a tensile stress state to the cells, since they are positioned far below the neutral axis of a generic 2D cross- 
section (see [1] for an analysis on the overall stiffness contribution of the various layers).

In the multi-scale approach, the in-plane displacements at the boundary of each cell are transferred to the micro-model, where a higher resolution of analysis is considered. Namely, the material microstructure of polycrystalline Si is taken into account and the progress of intergranular decohesion at grain boundaries is analyzed under plane stress conditions. Node matching at the cell boundaries for the macro-scale and micro-scale FE meshes is not necessary, since linear interpolation is used to project the boundary displacements from the regularly spaced macro-scale FE mesh to the non regular micro-scale mesh.

In the present modelling, it is implicitly assumed that the in plane displacements are responsible for cracking and that bending configuration of the cell can be neglected, so that the deformed configuration is confused with the undeformed one in the micro-model. As a result of these approximations, the principle of virtual work for the micro-model reads:

$$
\int_{V}(\boldsymbol{\nabla} \delta \boldsymbol{u})^{\mathrm{T}} \boldsymbol{\sigma} \mathrm{d} V-\int_{S} \delta \boldsymbol{g}^{\mathrm{T}} \boldsymbol{t} \mathrm{d} S=\int_{\partial V} \delta \boldsymbol{u}^{\mathrm{T}} \boldsymbol{f} \mathrm{d} S
$$

where the first term on the 1.h.s. is the classical virtual work of deformation of the bulk $V$ and the r.h.s. is the virtual work of the tractions acting on the boundaries of the cell $\partial V$. The second term on the l.h.s. is the contribution to the virtual work of the interface normal and tangential cohesive tractions $\boldsymbol{t}=(\tau, \sigma)^{\mathrm{T}}$ for the corresponding relative sliding and opening displacements $\boldsymbol{g}=\left(g_{\mathrm{T}}, g_{\mathrm{N}}\right)^{\mathrm{T}}$ at grain boundaries $S$. According to the cohesive zone model (CZM), tractions normal and tangential to the interface are opposing to the relative opening and sliding displacements of the grains evaluated at the interface level. In the present study, the Mixed Mode formulation by Tvergaard [10] is adopted, since it is suitable for modelling grain boundary decohesion in polycrystalline materials. The nonlinear equations relating the cohesive tractions to the normal and tangential relative displacements, $g_{\mathrm{N}}$ and $g_{\mathrm{T}}$, are:

$$
\begin{aligned}
\sigma & =\frac{g_{\mathrm{N}}}{l_{\mathrm{Nc}}} P(\lambda), \\
\tau & =\gamma \frac{g_{\mathrm{T}}}{l_{\mathrm{Tc}}} P(\lambda),
\end{aligned}
$$

where $P(\lambda)=27 \sigma_{\max }\left(1-2 \lambda+\lambda^{2}\right) / 4$ and $\lambda=\sqrt{\left(g_{\mathrm{N}} / l_{\mathrm{Nc}}\right)^{2}+\left(g_{\mathrm{T}} / l_{\mathrm{Tc}}\right)^{2}}$. The FE discretization of the micro-model is performed by using linear triangular elements for the grains and linear interface elements for the interfaces [11,12]. The discretized interface contribution in Eq.(19) becomes:

$$
\Delta \delta W_{\text {int }}=\delta \boldsymbol{u}^{\mathrm{T}} \boldsymbol{R}^{\mathrm{T}} \int_{S} \boldsymbol{B}^{\mathrm{T}} \boldsymbol{t} \mathrm{d} S
$$

where $\delta \boldsymbol{u}=\left[u_{1}, v_{1}, \ldots, u_{4}, v_{4}\right]^{\mathrm{T}}$ is the displacement vector, $\boldsymbol{R}$ is the rotation matrix 
of the interface element, and the matrix $\boldsymbol{B}$ contains its shape functions:

$$
\boldsymbol{B}=\left[\begin{array}{cccccccc}
-N_{1} & 0 & -N_{2} & 0 & N_{2} & 0 & N_{1} & 0 \\
0 & -N_{1} & 0 & -N_{2} & 0 & N_{2} & 0 & N_{1}
\end{array}\right]
$$

Due to the nonlinearity of the interface constitutive relation given by the CZM, the Newton-Raphson scheme is used, which allows to achieve a quadratic convergence in the computation. The reader is referred to [11] for more details about the computational issues. In this context, linearization of Eq.(22) yields:

$$
\Delta \delta W_{\text {int }}=\delta \boldsymbol{u}^{\mathrm{T}} \boldsymbol{R}^{\mathrm{T}} \int_{S} \boldsymbol{B}^{\mathrm{T}} \boldsymbol{C} \boldsymbol{B} \boldsymbol{R} \boldsymbol{u} \mathrm{d} S
$$

where $\boldsymbol{C}$ is the tangent constitutive matrix of the interface element containing the partial derivatives of the cohesive tractions w.r.t. the opening and sliding relative displacements [11.

In the micro-model, microcracking is originated by the in plane displacements passed as input from the macro-model (see Fig.11). This allows us to compute the updated stiffness of the Si cell, which coincides with that used in the macro-model at the first iteration only in absence of cracking. Moreover, the electrically inactive cell areas are determined from the inspection of the crack pattern. This updated information is passed back to the macro-model where the problem is solved again with the updated constitutive matrices. This procedure is iterated until convergence in the computed macro-displacement field is achieved. Since cracking affects only the Si cells, whose contribution to the overall stiffness of the plate element is small as compared to the the other layers, convergence is very fast.

This solution scheme implies the uncoupling between the microstructures of the Si cells analyzed in the micro-scale computations. This additional source of error, which is however limited by the fact that Si cells are not continuous but separated by EVA interlayers, is also minimized in the iterative convergence scheme. Regarding the computation of the tangent Young's modulus of the homogenized Si polycrystalline to be used in the macro-model computations, different techniques could be invoked. In the present study, the Young's modulus of Si is estimated by the ratio between the average stress and strain in the micro-model of the $\mathrm{Si}$ cell. The Poisson's ratio at the macro-level is not updated, since we do not expect significant variations due to microcracking.

\section{A numerical example}

In this section, a numerical example showing the applicability of the proposed numerical method to a realistic case study is proposed. The aim is the determination 


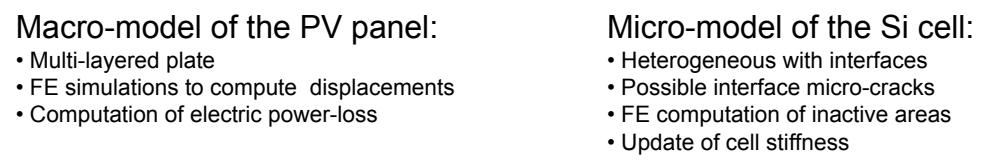

Micro-model of the Si cell:

- Heterogeneous with interfaces

- FE computation of inactive areas

- Update of cell stiffness

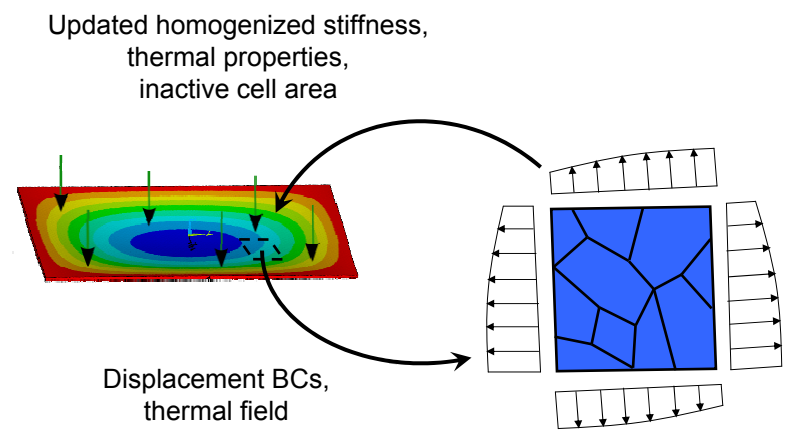

Figure 11. Iterative procedure involving the macro- and micro-models.

of microcracking in Si cells, the quantification of the electrically inactive cell areas and finally the computation of the $I-V$ and $P-V$ characteristics of the PV module. The comparison between the characteristics of the intact and microcracked modules will provide a measure of power-loss due to cracking.

A square PV module composed of $3 \times 3$ polycrystalline $\mathrm{Si}$ cells subjected to a uniform pressure $q=5400 \mathrm{~N}$ and simply supported along its sides is analyzed (see Fig.12 for the numeration of the Si cells). This type of load is recommended in the qualification standards to simulate the effect of a heavy snow. All the geometrical and mechanical parameters used for the plate elements in correspondence of Si cells and their separating domains are reported in Tab. 1 and 2, respectively. The flexural rigidity coefficient $K$ of the FE inside the cells is equal to $552746 \mathrm{Nmm}$, whereas in the separating domains is equal to $412138 \mathrm{Nmm}$. It is worth noting that the approximation of the PV panel as a homogeneous glass plate by disregarding the stiffening contributions of the layers below it, as considered in [4], leads to a flexural rigidity coefficient $K=409,135 \mathrm{Nmm}$. Such a value is considerably lower than the flexural rigidities used by accounting all the layers, with a consequent over-estimation of the displacements.

For the micro-model, the microstructure of the Si cells is obtained from the real image in Fig,2, Although a statistical variability of the grain size distribution and orientation takes place from a cell to another, we prefer here to use the same microstructure for all the cells. In absence of fracture anisotropy, the boundary cell displacements computed from the macro-model should induce the same crack pattern in the cells n. 1, 3, 7 and 9. The same reasoning applies to the cells n. 2, 4, 6 and 8. Hence, the comparison between different crack patterns in the various cells will put into evidence the role of the orientation of the grain boundaries.

The generation of the FE meshes for the micro-model is not a trivial task and 
Table 1

\begin{tabular}{|l|r|r|r|r|}
\hline Layer & $\begin{array}{r}H_{k} \\
(\mathrm{~mm})\end{array}$ & $\begin{array}{r}z_{k} \\
(\mathrm{~mm})\end{array}$ & $\begin{array}{r}E_{k} \\
(\mathrm{MPa})\end{array}$ & $\begin{array}{r}\nu_{k} \\
(-)\end{array}$ \\
\hline Glass & 4.000 & 4.000 & 73000 & 0.22 \\
EVA & 0.500 & 4.500 & 10 & 0.10 \\
$\mathrm{Si}$ & 0.166 & 4.666 & 130000 & 0.22 \\
EVA & 0.500 & 5.166 & 10 & 0.10 \\
Backsheet & 0.100 & 5.266 & 2800 & 0.10 \\
\hline
\end{tabular}

Geometrical and mechanical parameters for FE elements inside Si-cells (macro-scale model).

Table 2

\begin{tabular}{|l|r|r|r|r|}
\hline Layer & $\begin{array}{r}H_{k} \\
(\mathrm{~mm})\end{array}$ & $\begin{array}{r}z_{k} \\
(\mathrm{~mm})\end{array}$ & $\begin{array}{r}E_{k} \\
(\mathrm{MPa})\end{array}$ & $\begin{array}{r}\nu_{k} \\
(-)\end{array}$ \\
\hline Glass & 4.000 & 4.000 & 73000 & 0.22 \\
EVA & 1.166 & 5.166 & 10 & 0.10 \\
Backsheet & 0.100 & 5.266 & 2800 & 0.10 \\
\hline
\end{tabular}

Geometrical and mechanical parameters for FE elements inside the domain between two adjacent cells (macro-scale model).

a specific pre-processor developed in house is used. First, starting from a photo of the material microstructure, the grain boundaries are identified. Afterwards, all the grains are shrunk with respect to their center of mass and the interface nodes defining their polygonal geometries are duplicated. At this point, all the grains are meshed in their interior and interface elements are placed along the grain boundaries. The resulting FE connectivity matrices are given as input to the finite element analysis programme FEAP [13], where cohesive interface elements have been implemented by the first author [11]. The properties of the CZM are chosen to represent the fracture properties of an EVA-incapsulated Si $(E=169$ $\left.\mathrm{GPa}, \nu=0.22, \sigma_{\max }=190 \mathrm{MPa}, \gamma=1, l_{\mathrm{Nc}}=l_{\mathrm{Tc}}=0.0156 \mathrm{~mm}\right)$.

After applying the uniform pressure $q$ to the simply supported PV module, the macro-micro iterative scheme is applied and, at convergence, the macro and micro stress fields are computed. The contour plot of the macro-stress $\sigma_{x x}$ (the stresses in the horizontal direction $x$ ) in the PV module and the corresponding maximum micro-stress field in the central cell is shown in Fig.13. As expected, due to microcracking, the micro-stress field components are in general lower than the macro-stress ones, for the same applied cell boundary displacements.

The obtained microcrack pattern is shown in Fig.14. All the lines correspond to interface cracks with $\lambda>2.8 \times 10^{-3}$, which is a sufficient low value to select all 

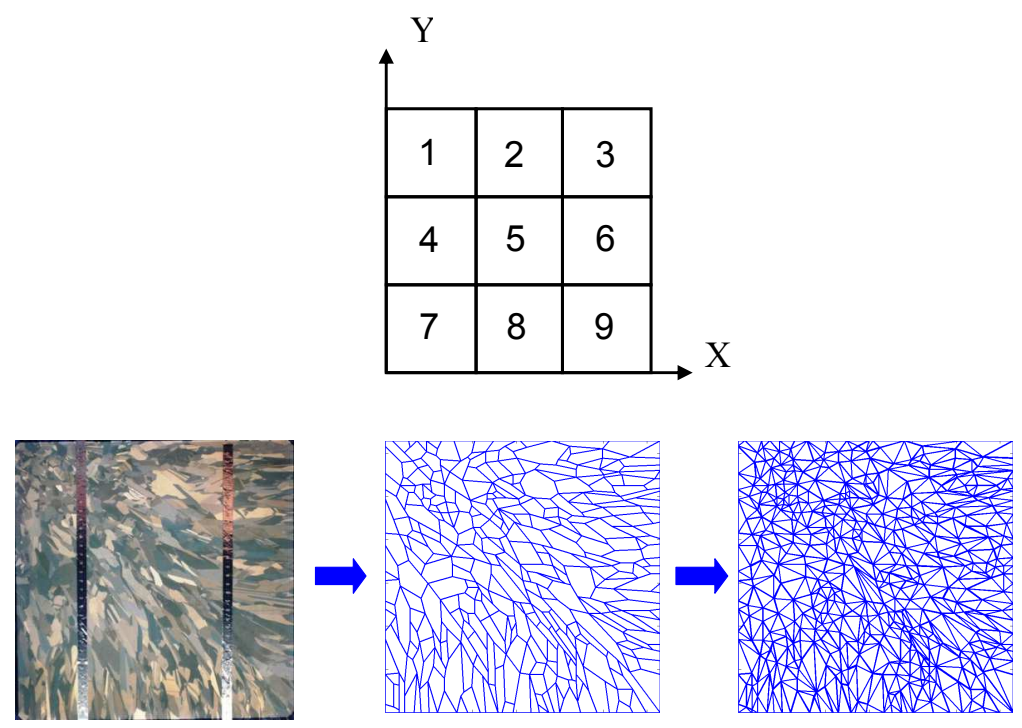

Figure 12. A PV module with $3 \times 3$ cells. Sequence of operations for the generation of the FE mesh of a Si cell for the micro-model: starting from a photo of the microstructure, the grain boundaries are identified and the FE mesh with interface elements is generated.

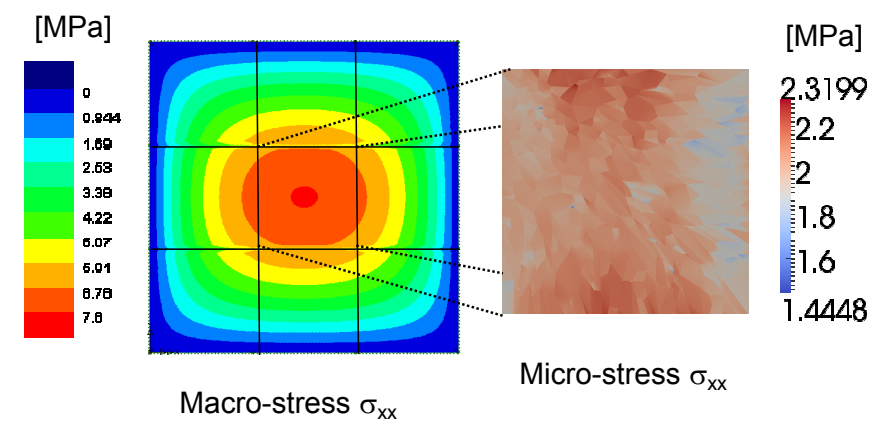

Figure 13. Macro- and micro-stresses in the horizontal direction.

the microcracks present in the system. However, none of them has $\lambda>1$, i.e., no stress-free macrocracks are present. This is consistent with the experimental evidence showing that, although no cracks can be observed with naked eyes, their effect on the electric performance of the module is quite relevant, as shown in Fig, 4 by the electrically inactive cell areas detected using the electroluminescence technique.

Particularly interesting is the analysis of the distribution of the orientation of the microcracks with respect to the busbars (shown with red lines in Fig 14). To do so, the absolute angle of inclination of the numerically detected microcracks, $|\vartheta|$, is computed and the corresponding frequency of occurrence is determined. The angle $|\vartheta|=0^{\circ}$ denotes cracks perpendicular to the busbars, whereas cracks with $|\vartheta|=90^{\circ}$ are parallel to the busbars. The latter lead to the highest electrically inactive cell areas and are therefore particularly harmful. 


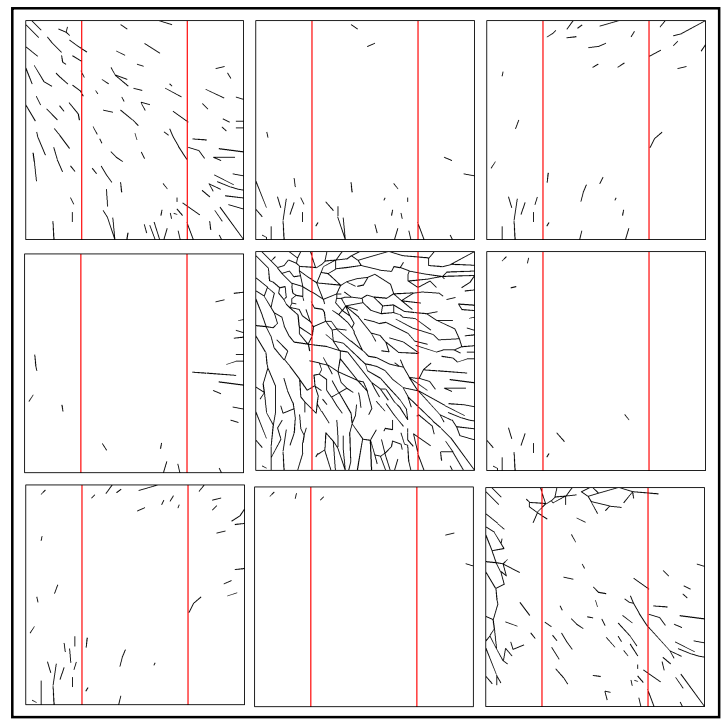

Figure 14. Computed crack pattern for a uniform pressure acting on the simply-supported PV module.

A comparison between the distribution of the orientations of microcracks inside the Si cells n. 5 (central cell) and 9 (lower right corner cell) is proposed in Fig.15(a), A quite uniform distribution is observed for the central cell, whereas a prevalence of microcracks with $|\vartheta|=45^{\circ} \div 60^{\circ}$ is observed for the corner cell. This is consistent with the experimental spatial and orientational distributions reported in 4], where it was noticed that microcracks tend to align perpendicularly to the direction of the maximum principal tensile strain. In the plate corners, the maximum principal tensile strain is orientated at $|\vartheta|=45^{\circ}$ and it promotes the opening of the corresponding interface microcracks.

However, in addition to the influence of the direction of the macroscopic strain field, the orientation of Si grain boundaries might also have a role on the intergranular crack distribution. This is shown in Fig 15(b), where the orientational distribution of microcracks in cells n. 7 (lower left corner) and 9 (lower right corner) are compared. Since the macroscopic strain field is basically the same for all the corner cells, the difference is solely ascribed to the different grain boundary orientations. The microstructure used for all the cells shows in fact an evident elongation of the grains from the down right corner to the upper left corner. As a result of this, the Si cell n. 7 is less prone to microcracking in the $45^{\circ}$ direction than the cell n. 9 .

The electrically inactive cell areas are also determined from the crack pattern in Fig,14 and are shown in Fig,16. According to the criterion illustrated in Fig,8, each cell is subdivided in three distinct regions: the first on the left of the first busbar, the second between the two busbars and the third on the right of the second busbar. For the first region, the inactive cell area is determined by the isolated cell area to the left of the skyline given by the ensamble of microcracks closer to the busbar. For the second region, the inactive cell area is represented by the isolated area between 

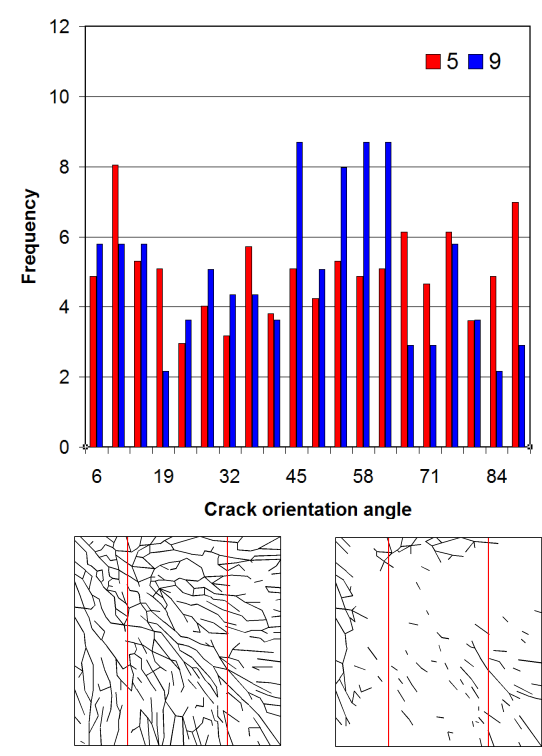

(5) Center

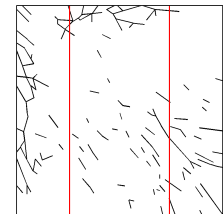

(9) Down right corner

(a) Central vs. down right corner cells

Figure 15. Distribution of the orientation of microcracks: comparison depending on the cell position. The angle $|\vartheta|=0^{\circ}$ denotes cracks perpendicular to the busbars (shown with red lines), whereas cracks with $|\vartheta|=90^{\circ}$ are parallel to the busbars.

two subvertical microcracks with the same vertical coordinates. Finally, for the third region, the inactive cell area is defined by all the cell areas to the right of the skyline given by the ensemble of microcracks closer to the busbar. This criterion should be considered as the worst case scenario, since it is implicitly assumed that all the microcracks are electrically insulated. Actually, partial conductivity in case of closure effects due to temperature variations are likely to occur and might be responsible for the oscillating electrical response of defective cells, as experimentally observed in [6]. According to the present assumptions, the damage variable for each cell can be computed as the ratio between the black and the whole cell areas. This can be done with a simple post-processing of the cracked Si cell images in Matlab by computing the amount of black and white pixels. The central cell is the most damaged with $D=79 \%$, whereas cells n. 1, 3, 7 and 9 have $D=32 \%, 12 \%$, $12 \%$ and $30 \%$, respectively. The less damaged cells are the n. 2, 4, 6 and 8, with $D=11 \%, 4 \%, 5 \%$ and $\sim 0 \%$, respectively. The damage variable for the whole PV module is equal to $D=79 \%$.

The electric response of the intact and microcracked PV modules $\left(a=2, I_{\mathrm{ph}}^{D=0}=\right.$ $8.3 \mathrm{~A}, I_{\mathrm{s}}^{D=0}=6 \times 10^{-5} \mathrm{~A}, R_{\mathrm{s}}=0.007 \Omega, R_{\mathrm{p}}=410 \Omega$ ) is finally compared in Fig.17. For the computation we also consider an irradiance $I_{r}=1000 \mathrm{~W} / \mathrm{m}^{2}$ and a working temperature $T=27^{\circ}(300 \mathrm{~K})$. The effect of microcracking is particularly evident and, in the present worst-case scenario, the power loss is particularly high, with a fill factor of the microcracked module reduced down to $15 \%$ from $65 \%$ of the initial 


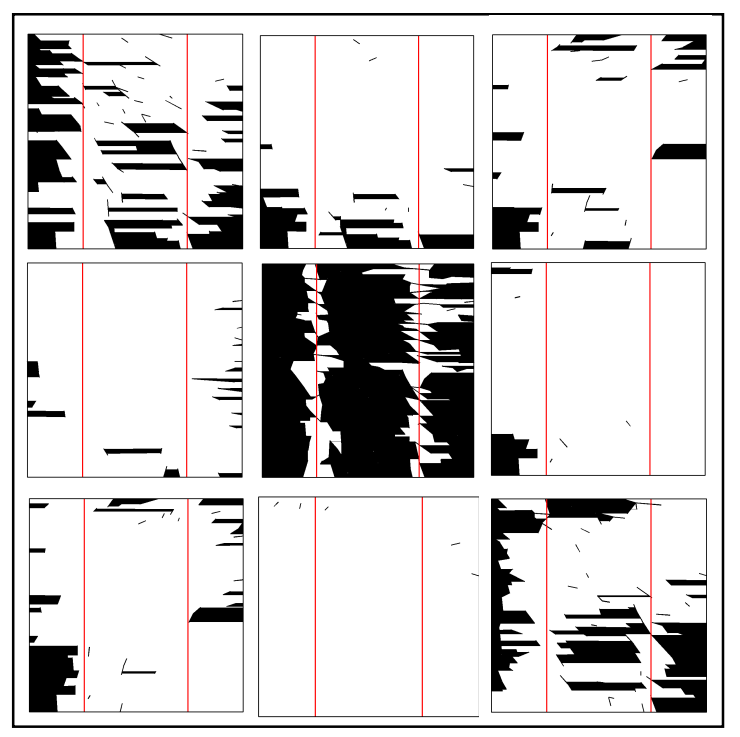

Figure 16. Computed electrically inactive cell areas according to the microcrack pattern in Fig 14 (worst-case scenario in case of electrically insulated microcracks).

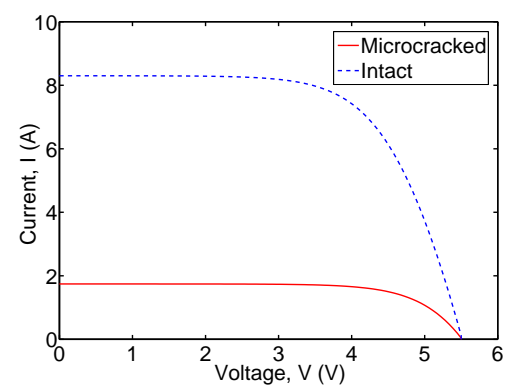

(a) Current vs. voltage.

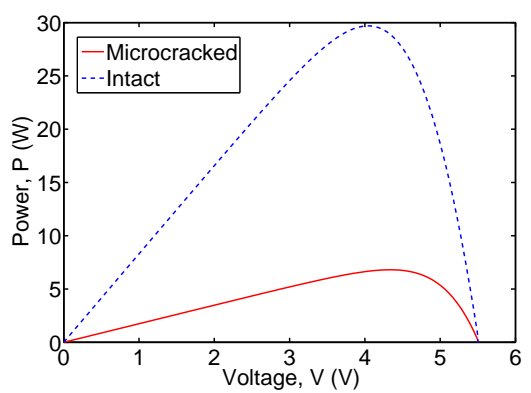

(b) Power vs. voltage.

Figure 17. Characteristic curves of the intact and micro-cracked PV module: current vs. voltage (a) and power vs. voltage (b).

one.

\section{Conclusion}

In the present work, a multi-physics and multi-scale (multi-resolution) computational approach has been proposed for the study of the snow load-induced microcracking in polycrystalline Si solar cells and its effect on the electric response of PV modules. To the authors' best knowledge, this is the first computational approach that attempts at studying the coupling between the elastic and the electric fields. Moreover, it is the first computational method that explicitly considers the polycrystalline grain microstructure of Si using a multi-resolution approach which permits to study the structural response of a PV module without neglecting the 
role of the grain boundaries as a source of microcracking. The numerical application shows that the proposed approach can be applied to realistic case studies.

Future research in this field will regard both the quantitative comparison with experimental measurements [14], parameter identification, and the development of additional computational features. In particular, the possibility of transgranular cracking, not modelled in the present study, will be analyzed. The coupling between the elastic and the thermal fields will also be put forward, in order to address the important issue of durability of PV modules exposed to cyclic temperature variations. The assumption of perfectly insulating microcracks will also be checked with ad hoc experiments and new constitutive micromechanical models for partially conducting microcracks will be implemented. The computationally efficient layerwise mixed theories for laminated plates proposed in [15] will also be considered in addition to 3D FE simulations.

\section{Acknowledgements}

The research leading to these results has received funding from the European Research Council under the European Union's Seventh Framework Programme (FP/2007-2013) / ERC Grant Agreement n. 306622 (ERC Starting Grant "Multifield and multi-scale Computational Approach to Design and Durability of PhotoVoltaic Modules" - CA2PVM). The support of the Italian Ministry of Education, University and Research to the project FIRB 2010 Future in Research "Structural mechanics models for renewable energy applications" (RBFR107AKG) is also gratefully acknowledged. MP thanks Dr. M. Köntges from the Institute of Solar Energy Research in Hamelin, Germany, for the useful discussion about the electrical model.

\section{References}

[1] M. Paggi, S. Kajari-Schröder, and U. Eitner. Thermomechanical deformations in photovoltaic laminates. Journal of Strain Analysis for Engineering Design, 46:772$782,2011$.

[2] A. Goetzberger, C. Hebling, and H.-W. Schock. Photovoltaic materials, history, status and outlook. Materials Science and Engineering, R, 40:1-46, 2003.

[3] S. Rein, K. Bothe, and B. Sattler. Qualitätssicherung und -Kontrolle in der Photovoltaikproduktion. FVS, BSW-Solar, 120-127, 2007.

[4] S. Kajari-Schröder, I. Kunze, U. Eitner, and M. Köntges. Spatial and orientational distribution of cracks in crystalline photovoltaic modules generated by mechanical load tests. Solar Energy Materials and Solar Cells, 95:3054-3059, 2011. 
[5] M. Köntges, I. Kunze, S. Kajari-Schröder, X. Breitenmoser, and B. Björneklett. The risk of power loss in crystalline silicon based photovoltaic modules due to microcracks. Solar Energy Materials and Solar Cells, 95:1131-1137, 2011.

[6] B. Weinreich, B. Schauer, M. Zehner, and G. Becker. Validierung der Vermessung gebrochener Zellen im Feld mittels Leistungs PV-Thermographie. In Tagungsband 27. Symposium Photovoltaische Solarenergie, Bad Staffelstein, Germany, pages 190196, 2012.

[7] J.A. Gow and C.D. Manning. Development of a photovoltaic array model for use in power-electronics simulation studies. IEEE Proceedings of Electronic Power Applications, 146:193-200, 1999.

[8] M.C. Alonso-Garcia and J.M. Ruiz. Analysis and modelling the reverse characteristic of photovoltaic cells. Solar Energy Materials and Solar Cells, 90:1105-1120, 2006.

[9] R. Szilard. Theory and Analysis of Plates: Classical and Numerical Methods. Prentice-Hall, Englewood Cliffs, New Jersey, Par. 3.7, 1974.

[10] V. Tvergaard. Effect of fiber debonding in a whisker-reinforced metal. Materials Science and Engineering A, 107:23-40, 1990.

[11] M. Paggi and P. Wriggers. A nonlocal cohesive zone model for finite thickness interfaces - Part II: FE implementation and application to polycrystalline materials. Computational Materials Science, 50:1634-1643, 2011.

[12] M. Paggi and P. Wriggers. Stiffness and strength of hierarchical polycrystalline materials with imperfect interfaces. Journal of the Mechanics and Physics of Solids, 60:557-571, 2012.

[13] O.C. Zienkiewicz and R.L. Taylor. The Finite Element Method. Fifth Ed., Butterworth?Heinemann, Oxford and Boston, 2000.

[14] S. Kajari-Schröder, Kunze I., and Köntges M. Criticality of cracks in PV modules. Energy Procedia, 27:658-663, 2012.

[15] E. Carrera. Evaluation of layer-wise mixed theories for laminated plates analysis. AIAA Journal, 36:830-839, 1998. 